\title{
Fluoride and sodium trimetaphosphate (TMP) release from fluoride varnishes supplemented with TMP
}

\author{
Michele Mauricio MANARELLI \\ Alberto Carlos Botazzo DELBEM \\ Célio PERCINOTO \\ Juliano Pelim PESSAN
}

Univ Estadual Paulista (UNESP), Araçatuba Dental School, Department of Pediatric Dentistry and Public Health, Araçatuba, SP, Brazil.

Declaration of Interests: The authors certify that they have no commercial or associative interest that represents a conflict of interest in connection with the manuscript.

\section{Corresponding Author:}

Juliano Pelim Pessan

E-mail: jpessan@foa.unesp.br

DOI: 10.1590/1807-3107BOR-2016.vol30.0064

Submitted: Dec 16, 2015

Accepted for publication: Jan 27, 2016

Last revision: Mar 08, 2016

\begin{abstract}
This study assessed fluoride (F) and sodium trimetaphosphate (TMP) release into artificial saliva from varnishes containing $0 \%, 2.5 \%$, and 5\% NaF, supplemented or not with 5\% TMP. The varnishes were applied on polyester sheets ( $n=8 /$ group), and F and TMP released into artificial saliva were measured for up to 24 hours. The amount of $F$ and TMP released were directly related to $\mathrm{NaF}$ and TMP concentrations in the varnishes. The highest $\mathrm{F}$ release was seen for $5 \% \mathrm{NaF}$ and $5 \% \mathrm{NaF}+$ $5 \%$ TMP, whereas 5\% TMP released the highest amount of TMP. However, the simultaneous addition of $\mathrm{NaF}$ and TMP to varnishes significantly reduced the amount of $\mathrm{F}$ and TMP released from the products.
\end{abstract}

Keywords: Fluorine; Polyphosphates; Sodium Fluoride.

\section{Introduction}

The addition of phosphate salts to topically applied fluoridated products has been proposed as a method to increase the anticaries effects of such formulations. Sodium trimetaphosphate (TMP) has recently been shown to promote a synergistic remineralizing effect both in vitro ${ }^{1}$ and in situ ${ }^{2}$ when added to fluoride (F) varnishes. However, considering the complex interactions between F and TMP and the scarcity of studies assessing the association between these compounds in varnish formulations, investigating the patterns of the release of $F$ and TMP from these products would be informative, as interactions between NaF and TMP salts, as well as among other varnish components, could potentially affect the efficacy of the products. Therefore, the present study assessed the release of $\mathrm{F}$ and TMP into artificial saliva from varnishes containing $\mathrm{NaF}$ and/or TMP over 24 hours.

\section{Methodology}

\section{Varnish formulation and fluoride assessment}

The varnishes were produced by SS White Dental Products (Rio de Janeiro, Brazil) and contained colophony, ethyl cellulose, tolu balsam, beeswax, toluene sulfonamide, vanillin, saccharin, and ethanol. F concentrations were $0 \%, 2.5 \%$, and $5 \%$ of NaF (Merck, Darmstadt, Germany), with or without the addition of TMP at concentration $5 \%(w / w)$ (Sigma-Aldrich Co., St. Louis, USA). A varnish without F and TMP (Placebo) and a commercial formulation (Duraphat ${ }^{\mathrm{TM}}$, Colgate, Colony, Germany) were also included. 
F concentrations in the varnishes were determined using an F ion specific electrode (9609 BN-Orion Research Inc., Beverly, MA, USA), coupled with an ion analyzer (Orion $720 \mathrm{~A}+$, Orion Research Inc.). F was recovered from the varnishes according to the protocol by Shen and Autio-Gold ${ }^{3}$ in triplicate. Samples were buffered using TISAB II prior to analysis.

\section{Release of fluoride and TMP from the varnishes}

The varnishes were applied on polyester sheets $(20 \times 40 \mathrm{~mm})$ (Mylar, Wilmington, USA), which were weighed before and after varnish application ( $n=8 /$ group). ${ }^{4}$ The sheets were then placed inside polystyrene vials containing $3 \mathrm{~mL}$ of artificial saliva $\left(1.5 \mathrm{mmol} . \mathrm{l}^{-1} \mathrm{Ca}\left(\mathrm{NO}_{3}\right)_{2} \cdot 4 \mathrm{H}_{2} \mathrm{O} ; 0.9 \mathrm{mmol}^{-\mathrm{l}^{-1}}\right.$ $\mathrm{NaH}_{2} \mathrm{PO}_{4} \cdot 2 \mathrm{H}_{2} \mathrm{O} ; 150 \mathrm{mmol} . \mathrm{l}^{-1} \mathrm{KCl} ; 0.1 \mathrm{~mol}^{-1} \mathrm{l}^{-1}$ Tris buffer; 0.03 ppm F; pH 7.0; unstirred, $\left.37^{\circ} \mathrm{C}\right) .{ }^{5}$ The sheets were transferred into new polystyrene vials containing fresh artificial saliva solutions at 30, 60, $90,120,180,240,300,360,540,720,900,1080$, and 1440 min after the first immersion. Amount of $\mathrm{F}$ released from the varnishes was determined, as previously described, after buffering with TISAB II. For TMP determination, the solutions were hydrolyzed with $1 \mathrm{~mol} / \mathrm{L} \mathrm{HCl}$, at a temperature just below boiling point. ${ }^{6}$ Total phosphorus was then determined using a colorimeter (EON Biotek, Winooski, USA). ${ }^{7}$

\section{Statistical analysis}

SigmaPlot software version 12.0 (SigmaPlot, Systat Software Inc., San Jose, USA) was used, with the significance level set at $5 \%$. Data obtained on the release of $\mathrm{F}$ and TMP, as a function of time, were analyzed by two-way, repeated measures analysis of variance (ANOVA) and Student-Newman-Keuls' test. Cumulative ion release data were analyzed by one-way ANOVA followed by Tukey's test.

\section{Results}

The mean (standard deviation) F concentrations ( $\mu \mathrm{g} \mathrm{F} / \mathrm{g}$ ) in the varnishes were 405.2 (84.9), 22,788.5 (2,553.1), 13,470.2 (911.2), 13,845.5 (703.5), 22,861.6 $(1,701.5), 405.6$ (74.8), and 21,187.6 (168.3), respectively for the Placebo, 5\% NaF, 2.5\% NaF, 2.5\% NaF/5\% TMP, $5 \% \mathrm{NaF} / 5 \% \mathrm{TMP}, 5 \% \mathrm{TMP}$, and Duraphat varnishes.
The time-course release of $\mathrm{F}$ and TMP from the varnishes into the artificial saliva is shown in Figures 1 and 2, respectively. The release of F was significantly influenced by the type of varnish $(\mathrm{F}=431.9, \mathrm{p}<0.001)$ and time $(\mathrm{F}=281.6, \mathrm{p}<0.001)$, with significant interaction between the variables $(F=38.0, p<0.001)$. Similarly, the release of TMP was influenced by the type of varnish $(\mathrm{F}=429.6, \mathrm{p}<0.001)$ and time $(\mathrm{F}=108.8, \mathrm{p}<0.001)$, with significant interaction between the variables $(\mathrm{F}=27.2, \mathrm{p}<0.001)$. The amount of $F$ released from varnishes without TMP was significantly higher than from those containing TMP, following a dose-response pattern according to the F content of the products. Significantly higher amounts of TMP were released from the 5\% TMP varnish compared with other formulations.

The statistical power calculated separately for the release of $\mathrm{F}$ and TMP from the varnishes over time was 0.916, considering the variables "varnishes" and "time", as well as the interaction between the two variables. The statistical power obtained for the cumulative release of $\mathrm{F}$ and TMP over $24 \mathrm{~h}$ was 0.958 .

\section{Discussion}

Different patterns for the release of $\mathrm{F}$ were observed for the TMP-containing varnishes, despite all products contained the amount of $F$ specified by the manufacturer. Therefore, there appeared to be inherent differences in the carrier for $\mathrm{NaF}$ in the $\mathrm{F}$ varnishes, which in turn affected the rate of ion released into the artificial saliva. Two factors seemed to have played an important role in the release of $\mathrm{F}$ from the products: varnish composition and TMP addition. Regarding the composition, the release of F from Duraphat was two-fold higher than that from the experimental varnish containing $5 \% \mathrm{NaF}$. As both these varnishes contained the same fluorinated salt $(\mathrm{NaF})$ and resin (colophony), the differences in the release of $\mathrm{F}$ between the two formulations may have been due to other ingredients and/or the ratios used in the manufacturing process. ${ }^{8}$ Regarding the presence of TMP, the total amount of $\mathrm{F}$ released from $2.5 \% \mathrm{NaF} / 5 \% \mathrm{TMP}$ and 5\% NaF/5\% TMP varnishes were $\sim 60 \%$ and $20 \%$ lower, respectively, than that from the varnishes without TMP, whereas the amount of TMP released from these formulations were $\sim 30 \%$ 

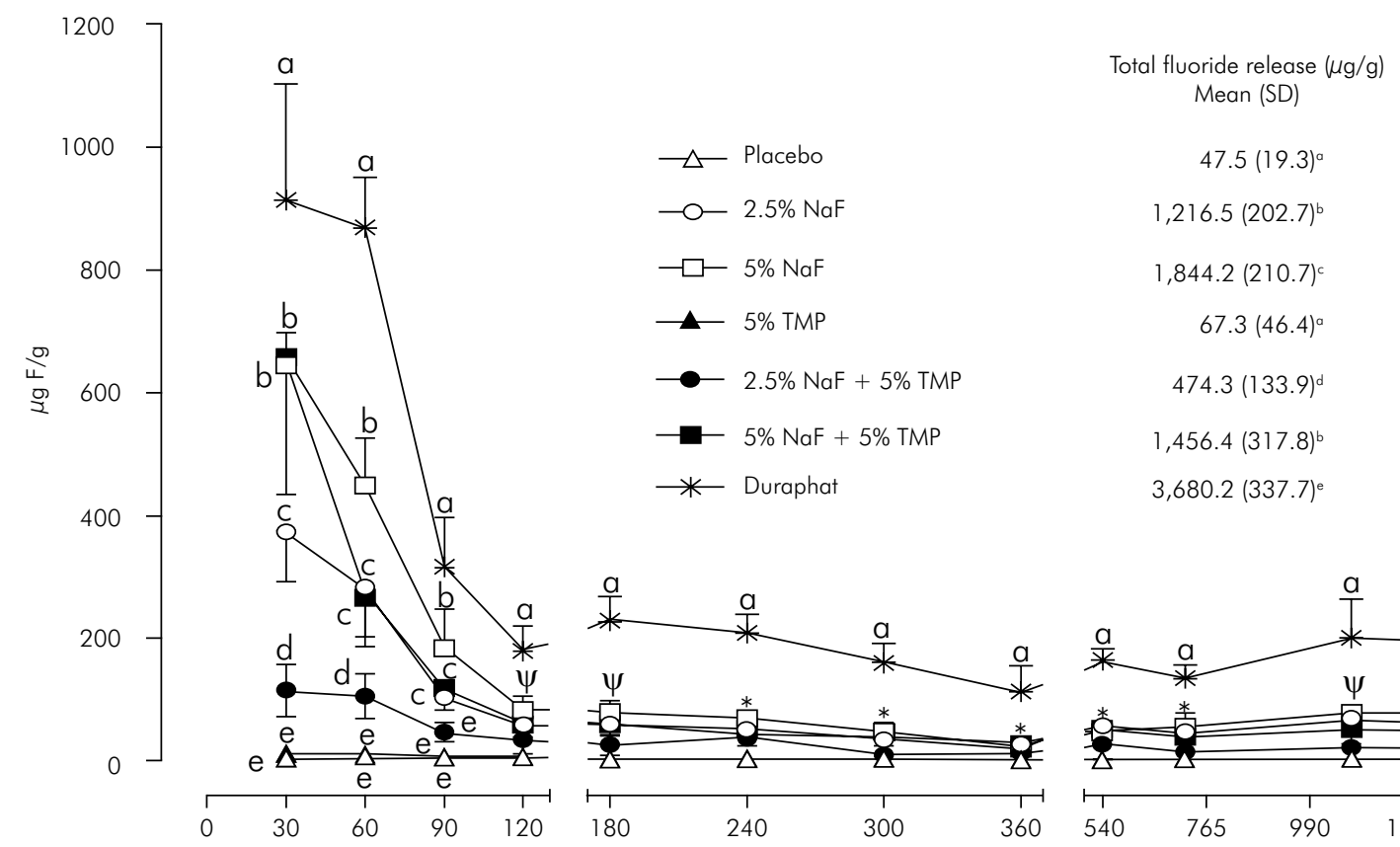

Figure 1. Time-course of the release of fluoride from varnishes into artificial saliva $(24 \mathrm{~h})$. Vertical bars indicate standard error of the mean. ${ }^{*} \mathrm{p}>0.05$. $\Psi \mathrm{p}>0.05$, except for $5 \% \mathrm{NaF} \times$ Placebo and 5\% NaF $\times 5 \% \mathrm{TMP}$ (two-way repeated measures-analysis of variance and Student-Newman-Keuls' test). Cumulative release of fluoride analyzed by one-way analysis of variance/Tukey's test $(p<0.05)$. Superscript letters indicate significant differences among the varnishes for each time point, as well as for cummulative data.

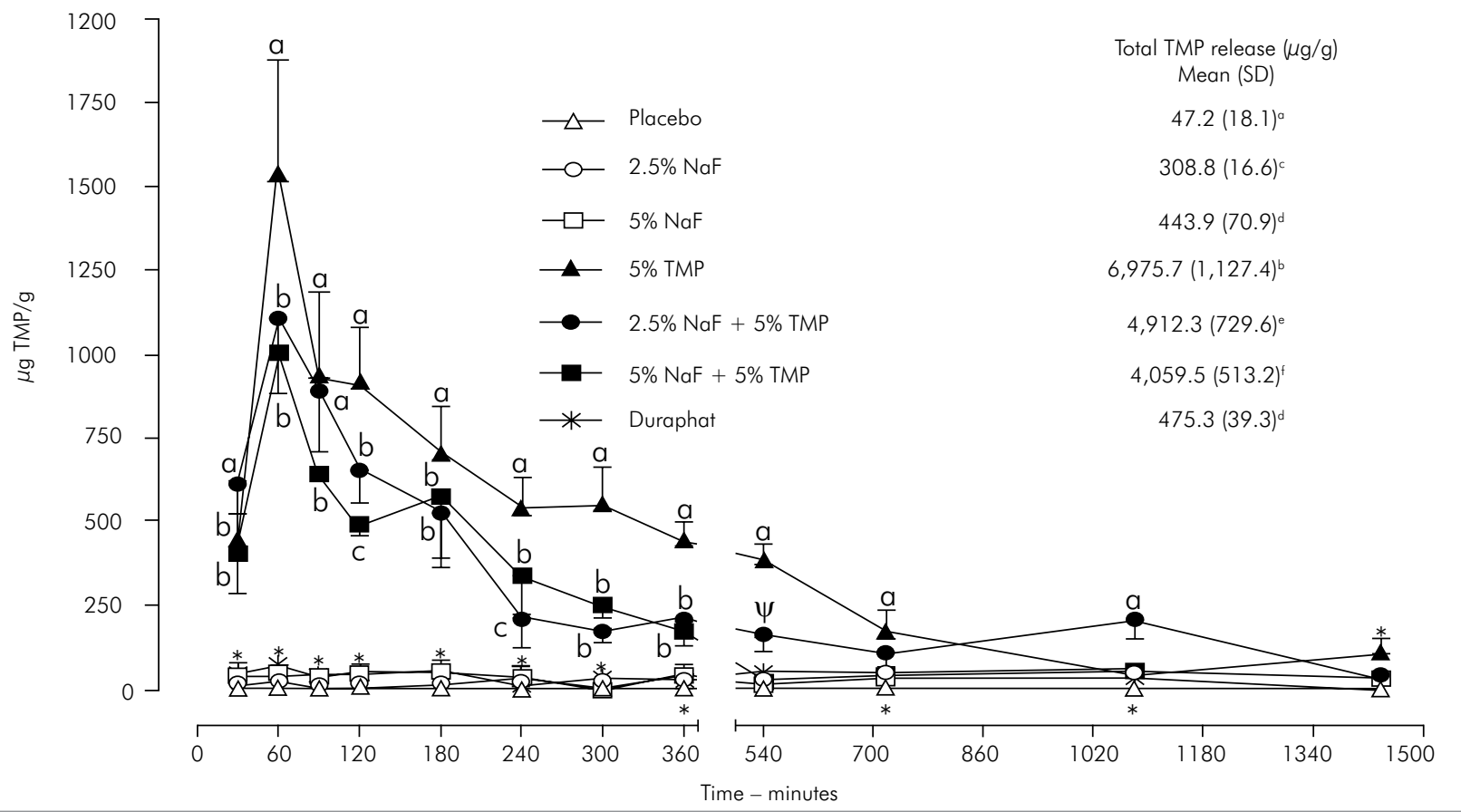

Figure 2. Time-course of TMP release from varnishes into artificial saliva $(24 \mathrm{~h})$. Vertical bars indicate standard error of the mean. ${ }^{*} p>0.05$. $\Psi \mathrm{p}>0.05$, except for $2.5 \% \mathrm{NaF} / 5 \% \mathrm{TMP} \times$ Placebo (two-way repeated measures-analysis of variance and Student-Newman-Keuls' test). Cumulative release of TMP analyzed by one-way analysis of variance/Tukey's test $(p<0.05)$. Superscript letters indicate significant differences among the varnishes for each time point, as well as for cummulative data. 
and $40 \%$ lower, respectively, than that released from the 5\% TMP varnish. Overall, these data indicate that the release of $\mathrm{F}$ and TMP are influenced by the appropriate TMP:F ratio in the product.

The partial and mutual inhibitory effect on the release of $\mathrm{F}$ and TMP observed when TMP and $\mathrm{F}$ were combined suggests a reduction in $\mathrm{CaF}_{2}$ deposition, which is in accordance with previous data on the same varnishes. ${ }^{1,2}$ However, such an effect was not proportional to the amount of $\mathrm{F}$ in the formulation, as different reductions in amount of $\mathrm{F}$ released from the $2.5 \% \mathrm{NaF} / 5 \% \mathrm{TMP}(\sim 60 \%)$ and $5 \% \mathrm{NaF} / 5 \%$ TMP ( 20\%) varnishes led to similar reductions in $\mathrm{CaF}_{2}$ deposition $\left.(\sim 57 \%)\right)^{1,2}$ Despite these reductions, the TMP-supplemented varnishes were shown to promote a significantly higher percentage of enamel surface hardness recovery and higher lesion body remineralization compared with the varnishes without TMP., ${ }^{1,2}$ Therefore, the present results, together with the effects on $\mathrm{CaF}_{2}$ formation and remineralization, suggest that the mechanism of action of TMP-supplemented varnishes follow a different pattern from that described for conventional varnishes. These appear to be related to the high affinity of TMP to enamel and intraoral calcium species, which are involved in the formation of more reactive remineralizing compounds after cariogenic challenges. ${ }^{1,2,9}$

\section{References}

1. Manarelli MM, Delbem ACB, Lima TMT, Castilho FCN, Pessan JP. In vitro remineralizing effect of fluoride varnishes containing sodium trimetaphosphate. Caries Res. 2014;48(4):299-305. doi:10.1159/000356308

2. Manarelli MM, Delbem ACB, Binhardi TDR, Pessan

JP. In situ remineralizing effect of fluoride varnishes containing sodium trimetaphosphate. Clin Oral Investig. 2015;19(8):2141-6. doi:10.1007/s00784-015-1492-6

3. Shen C, Autio-Gold J. Assessing fluoride concentration uniformity and fluoride release from three varnishes. J Am Dent Assoc. 2002;133(2):176-82. doi:10.14219/jada.archive.2002.0141

4. Manarelli MM, Moretto MJ, Sassaki KT, Martinhon CCR, Pessan JP, Delbem ACB. Effect of fluoride varnish supplemented with sodium trimetaphosphate on enamel erosion and abrasion. Am J Dent. 2013;26(6):307-12.

5. Moretto MJ, Magalhães AC, Sassaki KT, Delbem ACB, Martinhon CCR. Effect of different fluoride
The main limitation of the present study was that neither the dynamics of human saliva nor $\mathrm{pH}$ fluctuations in the oral environment affecting the rate of release were evaluated. Further, the precise amount of $\mathrm{F}$ and TMP required for caries prevention and the appropriate rate of release of F and TMP required for caries prevention after varnish application are unknown. However, the present data provide useful information regarding the pattern of release of $\mathrm{F}$ and TMP ions from the varnishes, and further investigations are required using protocols that better resemble clinical conditions.

\section{Conclusion}

The addition of TMP and $F$ to varnishes reduced the amount of $\mathrm{F}$ and TMP released from the formulations compared with products containing either TMP or $\mathrm{NaF}$.

\section{Acknowledgments}

The present study was supported by Coordenação de Aperfeiçoamento do Pessoal de Nível Superior - CAPES (scholarship to the first author). We also thankSS White Dental Products for manufacturing the varnishes. The second author holds a patent request for a product used in the study, by the Instituto Nacional da Propriedade Industrial - INPI, on 04/29/2008, no. 018080026091, PI0801811-1, published on January 11, 2011. concentrations of experimental dentifrices on enamel erosion and abrasion. Caries Res. 2010;44(2):135-40. doi:10.1159/000302902

6. Delbem ACB, Bergamaschi M, Rodrigues E, Sassaki KT, Vieira AEM, Missel EMC. Anticaries effect of dentifrices with calcium citrate and sodium trimetaphosphate. J Appl Oral Sci. 2012;20(1):94-8. doi:10.1590/S1678-77572012000100017

7. Fiske $\mathrm{CH}$, Subbarow $\mathrm{Y}$. The colorimetric determination of phosphorus. J Biol Chem. 1925;66(2):375-400.

8. Maas JRS, Faraco Junior IM, Lodi CS, Delbem ACB. Differences in loosely bound fluoride formation and anticaries effect of resin-based fluoride varnishes. Int J Paediatr Dent. 2013;23(3):166-72. doi:10.1111/j.1365-263X.2012.01240.x

9. Cochrane NJ, Saranathan S, Cai F, Cross KJ, Reynolds EC. Enamel subsurface lesion remineralisation with casein phosphopeptide stabilised solutions of calcium, phosphate and fluoride. Caries Res. 2008;42(2):88-97. doi:10.1159/000113161 